\title{
Ortopedia del alma. Degeneracionismo e higiene mental en la Casa de Corrección de Menores y Escuela de Trabajo San José, Colombia 1914-1947*1
}

Jairo Gutiérrez Avendaño*2

Lina Marcela Silva Ramirez*3

Objetivo: Comprender las prácticas y la formación experimental de la psicopedagogía de la infancia anormal en Colombia, llevadas a cabo en

150 la "Casa de Corrección de Menores y Escuela de Trabajo San José”, entre 1914 y 1947. Métodos y materiales: estudio cualitativo, de enfoque histórico-hermenéutico, mediante investigación documental, heurística de archivos de patrimonio documental e historiografia institucional del periodo de estudio. Conclusiones: el surgimiento de un nuevo régimen de educación especial (médico-psico-pedagógico) estableció un cambio relevante del modelo reformatorio de castigo al de intervención pedagógica, influida por el cruce de doctrinas cientificas de finales del siglo XIX y principios del XX, como la regla de lo normal y lo patológico, la teoría de la degeneración y la eugenesia, para el perfeccionamiento de un sujeto moderno capaz de ser útil por sí mismo y para la sociedad.

Palabras Clave: Corrección de menores, infancia anormal, degeneración, psicotecnia

${ }^{* 1}$ Este artículo se deriva del proyecto "Pobreza y locura como enfermedades sociales en la modernidad colombiana, 1850-1960" aprobado en la Convocatoria para la financiación de productos de investigación de menor cuantía 2014-2015 de la Fundación Universitaria Luis Amigó. Correo electrónico: jairo.gutierrezav@amigo.edu.co

*2 Fundación Universitaria Luis Amigó (Medelin, Colombia).

${ }^{*}$ Corporación Universitaria Minuto de Dios (Antioquia, Colombia). 


\section{HISTÓRIA DA PSIQUIATRIA}

\section{"Labrar el cuerpo y cosechar al hombre"}

Pensar la historia de las prácticas de corrección de los "menores díscolos" $\mathrm{y}$ de la "infancia anormal" a principios del siglo XX, permite conocer el surgimiento de una nueva sensibilidad frente a la situación social de una población emergente que debía regenerarse según la mentalidad civilizadora moderna, influida por el cruce de tres teorías: la identidad de lo normal y lo patológico; el degeneracionismo y la eugenesia. La primera, según Canguilhem (1983) fue definida como "Principio de Broussais", en los inicios de la fisiología de fines del siglo XIX, el cual estableció que la diferencia entre un extremo y otro se estimaba en su modificación cuantitativa y fue generalizada como un criterio estadístico o media ideal de tipologías físicas, médicas, psíquicas y morales, como lo concibió por su parte la doctrina de la degeneración del psiquiatra austro-francés Benedict Morel (1857), que tenía como tesis central que "los seres degenerados forman grupos o familias con elementos distintivos relacionados invariablemente a las causas que los transformaron en lo que son: un desvío mórbido del tipo normal de la humanidad".

El predominio de la propensión hereditaria como determinante de la degeneración, por sobre los factores ambientales, fue la base empírica de la eugenesia de Francis Galton que estableció el perfeccionamiento biomédico de la raza o especie humana, extendido a tres campos: la higiene física, mental y moral, la puericultura o cuidado materno-infantil, y a la homicultura para "labrar el cuerpo y cosechar al hombre". Galton cruzó métodos de las ciencias exactas y naturales, como la estadística y la biología, con la antropología y la psicología, de esta manera influyó en la creación de laboratorios de psicometría y antropometría, como en efecto funcionó en Colombia la primera Sección de Psicotecnia articulada con el área de fisiología en la Facultad de Medicina de la Universidad Nacional en la década del treinta. ${ }^{1}$ Es así como esta disciplina fue la base de la higiene mental y, a su

${ }^{1}$ En Colombia, Luis López de Mesa, reconocido integrante de academias nacionales, se especializó en Psicología Experimental y Psiquiatría en la Universidad de Harvard en 1917, se le atribuye la introducción del primer test psicológico, aplicado a una población de niños indigentes en Medellín - Antioquia, en los años veinte, y junto con la psicóloga española Mercedes Rodrigo Bellido y el fisiólogo Alfonso Esguerra Gómez, se reconocen como precursores de la psicotecnia, disciplina que dio origen a la psicología general - como también ocurrió en España, Brasil y Argentina - con la creación del Instituto de Psicología en la Universidad Nacional de Colombia entre 1947 y 1948 (Hernández, 2003, pp. 6-16). 
vez, del surgimiento de la psicopedagogía que tuvo como campo experimental los hospicios, las casas de anormales y de corrección de menores.

\section{Degeneracionismo, eugenesia e higiene mental}

"Corrige al niño y no castigarás al hombre" es la conocida sentencia pitagórica que asumió la pedagogía como corrección y está enraizada en la definición de "ortopedia", que viene del prefijo ortos, "correcto o derecho", unido al sufijo paidós, "niño" y paideia "educación", que significa: entrenar o educar corporalmente al niño para que crezca sin deformidades. Este sentido clásico cruzó de las técnicas de cuidado físico a las del alma, como se constata en el precepto Mens sāna in corpore sānō, retomado por la higiene como "saber vivir", ejercitada por diferentes tradiciones culturales de Occidente y que en una versión moderna se asumió como "higiene mental".

Se destaca que las ligas de higiene mental en España y Latinoamérica tuvieron en común su origen y funcionamiento en las asociaciones de neurología y psiquiatría, asimismo una estrecha relación de ambas sociedades con las

152 agencias de eugenesia, entre las décadas del veinte y treinta del siglo XX, según el registro de Sociedades e instituciones científicas para la América Latina de la Oficina Sanitaria Panamericana (Brasil, Chile, Cuba, Argentina, México, Perú, Uruguay) (1939, p. 38). De igual modo, cada una tuvo una sección de puericultura y, al mismo tiempo, tanto las sociedades de esta especialidad como de pediatría incluyeron una sección de eugenesia.

Un ejemplo de esta hibridación científica se constata en el caso de Colombia, donde se iba a realizar la Tercera Conferencia Panamericana de Eugenesia en 1938; pero, según Nancy Stepan (1991, p. 194), los organizadores decidieron sustituirla por "Congreso de Puericultura" el mismo año. Este cambio, más que retornar a dicha especialidad como un campo de la eugenesia, implicó una postura de distanciamiento frente al desprestigio de dicho término, extremado por la política médica nazi para el exterminio de la Lebensunwertes Leben: "vida indigna de ser vivida", que ejecutó la eutanasia a grupos de individuos que degeneraban la raza.

A finales del siglo XIX, entre las razones que motivaron la creación de las casas de corrección de menores en Colombia, se consideró a los delincuentes infantiles como parte del grupo de "anormales" y dentro del conjunto de "patologías sociales" ocasionadas por la degeneración de la raza.

Por su parte, el influjo de la Scuola Positiva Italiana o de antropología criminal, con Lombroso, Garófalo y Ferri, fue apropiada por médicos 


\section{HISTÓRIA DA PSIQUIATRIA}

colombianos como Luis Cuervo Márquez (1921, p. LXXXI), precursor de los primeros estudios de Medicina Social, junto con Carlos E. Putnam, fundador de la Oficina de Medicina Legal en el país, quienes se basaron en la teoría del "criminal nato", la cual clasificaba a los menores infractores como "criminaloides" una subdivisión de la categoría de "delincuente ocasional" planteada por Lombroso en su tratado L>uomo delinquente de 1876, para referirse a individuos con predisposición al delito, al cual llegaban por oportunidad e imitación. En ese sentido, se pretendía una equivalencia del delito como fenómeno natural prevalente en los "grados inferiores de la evolución humana" que para Lombroso eran los primitivos y los niños, relación que coincide con el carácter de "infantilización" y "primitivismo" de la raza atribuida en términos de retraso e incapacidad (sentido etimológico que comparte con "in-fancia", incapacidad de hablar o de responder) en la época del degeneracionismo en la primera parte del siglo XX.

En Colombia, la teoría de Morel fue apropiada por Miguel Jiménez López, a partir de su Cátedra Inaugural de Psiquiatría en la Facultad de Medicina de Bogotá, impartida en 1916, cuyo tema fue "La locura en Colombia y sus causas", según la cual esta se debía a una "viciosa" educación de la juventud, al alcoholismo y la sífilis. De esta manera, consideraba como causas ocasionales "la miseria nacional, violencia en la lucha por la vida, desproporción entre necesidades y medios de satisfacerlas, inconformidad de las clases sociales inferiores, importación de costumbres en decadencia (...)" (Jiménez, 1920). La Cátedra de Jiménez propició un debate convocado con el título de Los problemas de la raza en Colombia, en el Teatro Municipal de Bogotá en 1920, con siete conferencias unas a favor y otras en contra, que tuvieron un interés masivo y "espectacular" al punto de considerarlo "La mayor controversia científica de la intelectualidad colombiana".

La asimilación del degeneracionismo influyó en los planteamientos de Tomás Cadavid Restrepo ${ }^{2}$ — primer director de la Casa de Corrección de Menores y Escuela de Trabajo San José - en los que era frecuente la alusión a la anormalidad y la criminalidad infantil como una patología social, debida a factores como la herencia, la miseria, el alcoholismo y chichismo (consumo de bebida fermentada de maíz), la locura, la sífilis, la mala educación, entre otros.

${ }^{2}$ Nació en Medellín - Antioquia (1883-1952). Abogado de la Universidad de Antioquia. Estudió Sociología, Pedagogía y Filosofía en la Sorbona y en la Universidad Católica de Paris. Integrante de la Liga Internacional de la Educación Nueva, fundada en Bélgica en 1921, por un grupo de pedagogos de diversos países: Decroly en Bélgica; Dewey en Estados Unidos, Piaget, Cleparéde y Ferriére en Suiza, entre otros; y en Colombia, junto con Agustín Nieto Caballero y Rafael Bernal Jiménez. 
Así lo expresó el director en la prensa de la época sobre los menores díscolos: "de herencias malditas de padres desconocidos, hijos del infortunio y del hambre, expuestos a dolores y crueldades ajenas. Muchos son enfermos congénitos, otros adquieren sus males al contacto con sus compañeros de infortunio" (Cadavid, 1920, pp. 155-156). Estas causas heredo-degenerativas también fueron atribuidas por parte de los médicos de la Casa Correccional de Paiba, en el departamento de Cundinamarca (Bejarano y Sanmartín, 1923).

\section{"Discolía de la pubertad"}

El niño díscolo o "difícil" (del griego dyskolos) en Colombia fue objeto de estudio y de intervención atribuido a la pedagogía social, integrada por saberes como la psicología, biología, estadística, higiene, entre otros. En efecto, Cadavid refiere que era imprescindible la explicación etiológica y una terapéutica de la "discolía de la pubertad" con base en la Patología General de Paul Courmont (1913), al considerar que así como la terapéutica "será patogénica o no será", de igual modo dice Cadavid que la pedagogía "será psicológica o no será" (Cit. Cadavid, 1924, p. 19).

En ese postulado patológico, se localizó en la pubertad (entre los 10 y 15años de edad) la aparición de la psicosis juvenil o hebefrenia, asociada con la "demencia precoz" (ancestro de la nosografía de esquizofrenia), junto con la criminalidad como desorden de la conducta. Esta hipótesis Cadavid la respaldaba con estadísticas del aumento de ingresos de menores de edad al Manicomio entre 1917 y 1923.

La justificación de este saber "higiénico-pedagógico", como lo definió Cadavid, se debía al desconocimiento que tenían los educadores, padres de familia y autoridades sobre las causas y tratamiento de la discolía de los adolescentes, a quienes se sometía al castigo y a la represión por mano propia o bajo el régimen de reformatorios, a donde podían ser remitidos para internamiento voluntario por sus familias. La principal terapéutica para dicho estado "semi -patológico" era la actividad al aire libre, cultivo de la tierra, artes y oficios que garantizaran una habilidad técnica para la vida; alimentación balanceada, higiene mental para dosificar el trabajo escolar, y educación física y sexual.

\section{Clasificar y separar: del régimen punitivo al pedagógico}

La disposición del castigo como corrección se planteaba, por ejemplo, en la visión modernizadora de la élite del municipio de Medellín, que en la mentalidad 


\section{HISTÓRIA DA PSIQUIATRIA}

de Ricardo Olano, uno de sus principales promotores, en 1917 expuso la necesidad de crear una "verdadera casa de escarmiento" para:

(...) formar ciudadanos obedientes, prevenidos del libertinaje y la homosexualidad dentro de los imperativos de la moral católica (...) en donde se pueda vigilar, dormitorios repartidos a lo largo de los salones (...) en donde los trabajos impuestos como castigos, sirvan de penitencia, de medio de conversión y de corrección (...) donde se pueda emplear el látigo, los reglazos y el calabozo sin oídos y miradas impertinentes. Por supuesto un lugar en donde se instruya, se capacite y viva en el temor de Dios. (Olano, 28/03/1917)

Para esta actitud contra la decadencia de la raza, los menores díscolos y anormales eran considerados "un lumpen de la naturaleza humana sobre el que se ha proyectado tradicionalmente desde las visiones más fatalistas, hasta las más ardientes empresas pedagógicas repletas de simbolismos regeneracionistas - cuando no directamente 'redentores' - , desde el etiquetado nosográfico más aparentemente aséptico, hasta la beneficencia y la educación especial" (Huertas, 1998, p. 9).

El origen de un nuevo régimen de educación especial (médico-psico-pedagógico), en Colombia se enmarca en el contexto de finales del siglo XIX, según Juan Díaz (1987, p. 17), época en que la ciudad de Medellín, capital del departamento de Antioquia, inició su industrialización y crecimiento urbano en medio de problemáticas sociales de todo tipo como la falta de vivienda, barrios marginados, incremento de delincuencia juvenil, prostitución, abandono de menores y el trabajo de la madre fuera de casa; dichas situaciones motivaron una sensibilidad filantrópica, de beneficencia pública y protección social de la infancia.

La mayor prioridad era clasificar y separar a los niños infractores de la población adulta, con la que se encontraban recluidos en establecimientos carcelarios. De esta situación da cuenta el abogado Miguel Martínez, en su estudio de Criminalidad y Violencia en Antioquia (1895/1992), el cual denunciaba que "los presidios y casas de reclusión no son edificios construidos para este fin mal ventilados, desaseados, oscuros - nunca a este respecto se ha cumplido el mandato de la ley (...) en estos lugares hay reunión de niños y jóvenes inexpertos con criminales avezados" (Martínez, 1895/1992, p. 38).

Una serie de informes de la dirección de la cárcel de varones, de 1911 y 1914, consignaban que "con frecuencia conducen a la cárcel a niños de menor edad, los que forzosamente tienen que rozarse con la generalidad de los presos... puede garantizarse que en lugar de conseguir reforma, adquieren mayores conocimientos del mal y salen preparados para delinquir" (Gobernación, 1912, p. 97).

Debido a esta situación, el alcaide de la Cárcel de Varones de Medellín, propuso la creación de una casa de corrección de menores, porque "a pesar del celo desplegado para evitar el trato de los menores con los demás presos, no es 
posible conseguirlo del todo. Es menester hacer una separación absoluta y para ello convendría detenerlos en otro lugar distinto al de la cárcel, para que no adquieran nuevos vicios (...)" (Gobernación, 1914, pp. 99-100). Igual ocurría en la misma época, de la primera mitad del siglo $\mathrm{XX}$, con las frecuentes quejas sobre niños y niñas menores de doce años internos en los manicomios expuestos al contacto con "dementes" adultos, en los departamentos de Cundinamarca (Brigard, 1924, p. 23), Antioquia (Uribe, 1940, pp. 104-112) y Valle del Cauca (Relator, 12/02/1930).

La Casa de Corrección y Escuela de Trabajo San José se creó por Ordenanza No. 5 del 17 de marzo de 1914, sancionada por la Asamblea Departamental de Antioquia, la cual estableció que los menores admisibles eran:

1) los condenados a prisión, arresto o trabajo en obras públicas, 2) los recluidos por infracción de las disposiciones de policía, 3) los moralmente abandonados y que no tengan persona capaz que los reclame para su custodia y educación, 4) los concentrados por voluntad de sus padres o tutores, y los que por vía de amparo envíen las autoridades respectivas.

Una vez efectuada esta clasificación administrativa, Cadavid estableció una triple división entre los anormales: 1) físicos (gigantismo, infantilismo, macrocefalia, formas asimétricas del cráneo, etc.), estos se definieron como hiposténicos o débiles de fuerza y tono muscular, e hiperesténicos o de proporciones corporales excesivas. 2) intelectuales (idiocia, imbecilidad, debilidad mental), esta última se clasificó en dos categorías de atrasados (arriérés): los deprimidos o perezosos y los inestables o indisciplinados. 3) morales (extrínsecas, perversiones sexuales y difíciles) (Cadavid \& Velásquez, 1921, p. 14).

Según el referido informe que Cadavid presentó como director de la Casa (1921), así como su estudio sobre Discolía de la pubertad (1924), la deficiencia mental fue intervenida por métodos experimentales de la pedagogía activa de Ovide Decroly (1923) del que adoptó la clasificación de anormales "sensoriales, motores y afectivos"; la psicopedagogía de Alfred Binet y Theodore Simone $(1917)^{3}$, y de Jean-René Cruchet ${ }^{4}$ (1908), disciplinas que en la Casa tuvieron por

${ }^{3}$ El Test de Inteligencia de Binet y Simon, empleado en la Casa de Menores y Escuela de Trabajo, consistía en medir atributos del cráneo, fuerza física, y una serie de treinta tareas de complejidad creciente, para determinar la "edad mental" del niño y, así, el nivel de escolaridad que debía ocupar en el sistema educativo.

${ }^{4}$ J-R Cruchet en su texto Les arriérés scolaires (1908), define que el niño con retardo escolar "es todo niño que desde el punto de vista escolar está retrasado 2 o 4 años en relación a la media escolar de los niños de su edad", citado por Cadavid en su Informe de la Casa de Menores (1924, p. 6). 


\section{HISTÓRIA DA PSIQUIATRIA}

objeto clasificar y corregir como principales acciones psicopedagógicas de los menores difíciles.

Raymond Buysé, ${ }^{5}$ en su Estudio crítico sobre los orígenes de la pedagogía moderna, conferencia presentada en la Facultad de Educación de la Universidad Nacional de Colombia en 1933, se refiere a la genealogía que dio origen al movimiento de la "psicología experimental", así como su incidencia en el movimiento de pedagogía activa para la educación de anormales. De esta manera Buysé recurre a la síntesis que Van Biervliet ${ }^{6}$ reseñó en su texto Premiers éléments de pédagogie expérimentale (1911), traducido al castellano como Pedagogía experimental (1925), para uso de las escuelas normales:

a) la raíz patológica, que consiste en el esfuerzo de los médicos alienistas, psiquiatras (Charcot), neuropatólogos (Grasset), criminalistas (Lombroso). b) La raíz antropológica, que comprende los trabajos de los frenólogos (Gaal), de los embriólogos (hechos de la herencia), de los antropólogos (Broca, localizaciones cerebrales). c) La raíz psicológica que comprende las investigaciones de Galton, Cattel, Binet, de Stanley Hall, de Meumann, etc. d) La raíz pedagógica, en la cual se incluyen las investigaciones concernientes a la educación de los anormales (Itard, Seguin), el estudio objetivo de los problemas pedagógicos, no solamente para conocer la naturaleza de los fenómenos conscientes puestos en juego, sino las diferencias susceptibles de registrarse; no las cualidades de las funciones mentales, sino más bien su rendimiento (Buysé, 1933, pp. 139-140).

El establecimiento de una tecnología de vigilancia del alma y corrección del cuerpo constituyó la "ortopedia escolar" de la Casa de Corrección de Menores y Escuela de Trabajo San José, esta asumía a los niños anormales y atrasados según la definición de Binet y Simón (1917): "los que no son admisibles en escuela ordinaria ni en hospital; parécele a la escuela poco normales, no los halla el hospital bastante enfermos" (Binet \& Simon, 1917). Para Cadavid, esta última la consideró imprecisa porque no era causal, por lo tanto, optó por la evidencia frenopática del estigma "los que tienen algún defecto importante en el cuerpo o en el alma". De hecho, Morel dio un papel relevante a las cachets typiques o

${ }^{5}$ Raymond Buysé, profesor de Pedagogía Experimental en la Universidad de Lovaina y colaborador de Decroly. Fundador del Laboratorio de Pedagogía Experimental de Lovaina, Doctor en Ciencias paidológicas, coautor, junto con Decroly, de un manual sobre test mentales (Los test mentales. Aplicaciones de la psicología a la organización humana y a la educación en los Estados Unidos) (Sáenz, Saldarriaga y Ospina, 1997, Vol. 1, p. 82)

${ }^{6}$ Jules Jean Van Biervliet, profesor de Psicología experimental en la Universidad de Gante, Bélgica. Miembro de la Academia Real Belga. 
stigmatas, que son marcas o signos de degeneración que caracterizan a las diferentes familias y grupos de degenerados (Morel, 1857, p. 37).

Por otra parte, de acuerdo con Simon y Decroly, Cadavid consideraba que debía sustituirse la noción de "pedagogía de anormales" por la de "niños de educación difícil", por tratarse de un concepto demasiado impreciso y despectivo. En la Casa se clasificaron los menores en dos grupos: 1) los atrasados (arriérés), intelectual y escolarmente asociados a los anormales, y 2) los perturbados de carácter, los inestables e indisciplinados, desubicados desde el punto de vista escolar (Cadavid \& Velásquez, 1921, p. 17).

Como lo dispuso dicho mandato de fundación, en la Casa se creó la sección especial de "incorregibles", para aquellos menores que después de una permanencia en la institución, suficiente para comprobar que eran incorregibles, se remitieran a la cárcel, penitenciaría, colonia penal, o manicomio, según el caso.

Antes de la reforma, la Casa de Menores y Escuela de Trabajo tenía un carácter de correccional basado en los modelos de reformatorios de Europa y Estados Unidos, en particular tuvieron como referente a la Colonie agricole et pénitentiaire pour les jeunes contrevenants de Mettray en Francia (creada en 1839), según se cita en la Ley 123 de 1890, "sobre casas de corrección de menores y Escuelas de Trabajo" se pretendía que "la construcción, el personal y el reglamento debían ajustarse hasta donde la situación, necesidades, costumbres y medios pecuniarios de Colombia lo permitieran" (Congreso de la República, 1890, pp. 378-379); de hecho, según Foucault, dicha colonia constituyó un precedente que abrió una nueva época en las tecnologías disciplinarias y de control, en tanto que concentró en un mismo espacio los modelos familiar, militar, escolar y judicial (2003, pp. 330-301).

La exposición de motivos del proyecto de creación de la Casa de Menores y Escuela de Trabajo San José, se enmarcó en el funcionamiento de Mettray y del Elmira Reformatory, Chemung Conty - New York (fundado en 1876), el cual se caracterizó por emplear métodos psicológicos para la regeneración de los menores, más que los medios punitivos, del mismo modo que se buscó implementar en Colombia en la primera mitad del siglo XX (Gobernación de Antioquia, 1920 , p. 3). 


\section{HISTÓRIA DA PSIQUIATRIA}

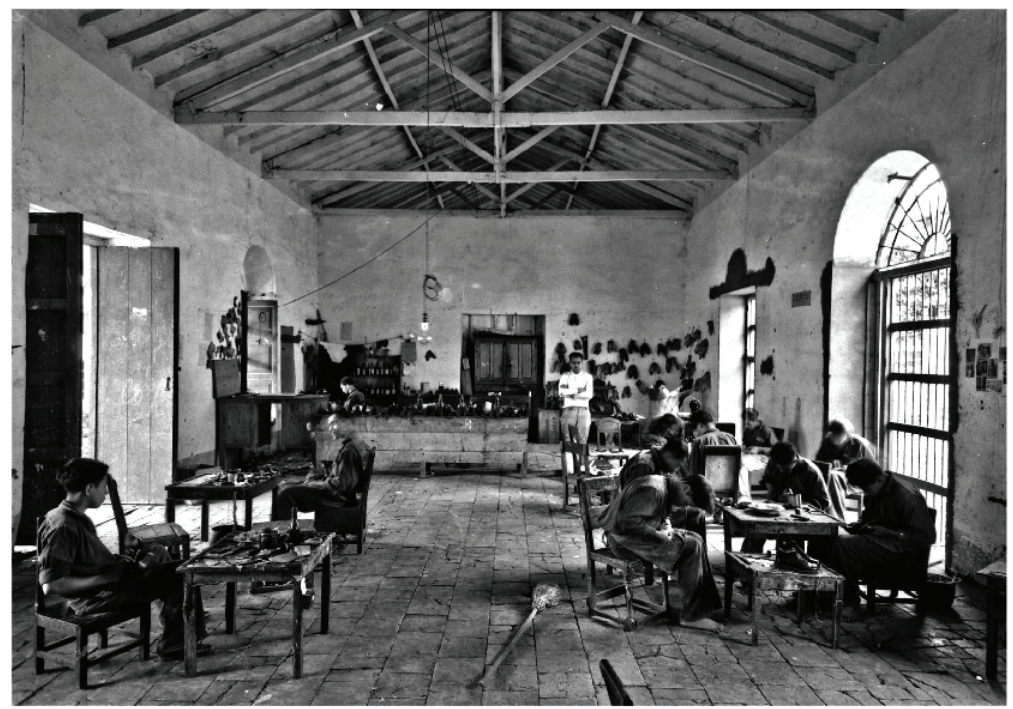

La Casa de Menores y Escuela de Trabajo tenía como fundamento reformatorio la enseñanza de artes y oficios, como la zapatería, para la formación de jóvenes productivos a la sociedad y el autosostenimiento del establecimiento. Foto: Francisco Mejía. Archivo Fotográfico Biblioteca Pública Piloto de Medellín, Código BPP-F-004-0628.

\section{Un "laboratorio de almas"}

La Casa estableció un régimen panóptico, según los directores Cadavid y Velásquez (1921), en términos de "auscultar", "acechar", "descubrir", "detectar", "investigar" el estado oculto de la anormalidad del niño mediante técnicas de observación permanente. Estos asumieron la labor del maestro psicopedagogo como un "arte de corrección y reforma" en metáforas escultóricas como las de pulir, moldear, tornear, propias de un "tallador de almas" (Cadavid \& Velásquez, 1921, p. 24). De hecho, la Casa de Menores fue definida por Cadavid como "un taller de regeneración moral y material". Así, el objetivo de esta era reformar "un hombre apto para la lucha y capaz de ser en la sociedad una unidad utilizable" (1921, p. 23).

En efecto, uno de los jurados que comentó el estudio Discolía de la pubertad de Cadavid, se refiere a un nuevo campo de saber que busca "penetrar el fondo de las almas, sin olvidar que su envoltura es de barro y que sus resonancias 
interiores no pueden ser desatendidas por los encargados de modelar su existencia" (Toro, 1924, p. 3). En ese propósito, se consideró que la escuela debía ser un "laboratorio de almas" (Cadavid, 1924, p. 57) para realizar pruebas psicotécnicas que permitieran evaluar las capacidades humanas. Sin embargo, el director Cadavid advertía que la psicología escolar era una ciencia muy reciente en el medio colombiano y que, por tanto, no se podían precipitar en dar una exactitud cuantitativa a los resultados psicométricos, porque "se sabe que los actos del espíritu no pueden someterse a medida matemática, digamos como el área de un terreno, no; lo que se valora es el fenómeno que acompaña al acto psíquico" (Cadavid, 1924, p. 31). Según este planteamiento, era fundamental cruzar dichos datos con la observación clínica, la experiencia de vida y los antecedentes familiares del niño.

\section{Decadencia del régimen reformatorio}

De acuerdo con lo expuesto, la coexistencia de prácticas de corrección, tecnologías psicopedagógicas y biomédicas, estuvo afianzada por los ideales de formación de un nuevo sujeto moderno que buscaron establecer acciones para impedir la reproducción de las desviaciones de la ley natural transpuestas al orden social.

No obstante, luego de casi tres décadas de su fundación, en una entrevista a Cadavid para la revista Raza en 1948, decía:

De esta obra maravillosa no ha quedado ni la sombra. No fue suficiente que el espíritu revolucionario de Barrios Ferrer, le diera al reformatorio un impulso de carácter científico, ni que la brillante rectoría de Tulio Gaviria, lo elevara a la altura de las mejores correccionales por su disciplina de responsabilidad. Hoy estamos en capacidad de afirmar que aquella casa, en vez de ser un laboratorio de rehabilitación humana, es una prisión de características horrendas. (Bravo, 1948, pp. 6-7)

Aunque la Casa de Corrección de Menores y Escuela de Trabajo San José fue un dispositivo en el que se hicieron visibles los ideales del progreso, vinculados al perfeccionamiento de la raza y el cultivo de ciudadanos modelados a la moral católica que regulaba un modo de vida "cristiana y en policía"; la propuesta de pedagogía activa de Cadavid se enfocó en la importancia de comprender científicamente los factores que determinaban la atención al menor difícil y anormal, razón por la cual sus aportes generaron una pauta para su reconocimiento en el ámbito psicopedagógico y sociojurídico. 


\section{HISTÓRIA DA PSIQUIATRIA}

\section{Referencias}

Asamblea Departamental de Antioquia (1914). Ordenanza Nº5 de 17 de marzo de 1914, sancionada por el Señor Gobernador de Antioquia Carlos Cock, se creó la "Casa de Corrección y Escuela de Trabajo". Medellín: Imprenta Oficial, p. 2.

Bejarano, J. \& Sanmartín, R. (1923). La situación de la Cárcel de Menores de Paiba. Informe. El Tiempo, 12 de diciembre 1923.

Binet, A. \& Simón, T. (1917). Niños anormales. Barcelona: Librería y Tipografía Médicas. Cit.: Cadavid, T.; Velásquez, D. (1921). Informe de la Casa de Menores y Escuela de Trabajo. Medellín: Imprenta Oficial. Sala Antioquia U.de.A.

Bravo, L. (1948, julio). La vida ejemplar, docta, sencilla del maestro Tomás Cadavid Restrepo. Revista Raza, 4(22), 6-7.

Brigard, L. (1924). Informe del Sindico de los Asilos de Indigentes y Mendigos ante la Junta General de Beneficencia de Cundinamarca. Bogotá: Casa Editorial de La Cruzada. p. 23.

Buysé, R. (1933). Estudio crítico sobre los orígenes de la pedagogía Moderna I. Revista Educación. Facultad de Educación Universidad Nacional. 1(3), 134-146. In Yarza, Alexander (2011). Preparación de maestros, reformas, pedagogía y educación de anormales en Colombia: 1870-1940 (Tesis de Maestría en Educación). Medellín: Universidad de Antioquia, pp. 209-210.

Cadavid, T. (1920). Casa de Menores. El Correo Liberal, abril 19, pp. 155-156.

Cadavid, T. \& Velásquez, D. (1921). Informe de la Casa de Menores y Escuela de Trabajo. Medellín: Imprenta Oficial. Sala Antioquia U.de.A.

Cadavid, T. \& Velásquez, D. (1924). Discolía de la pubertad. Medellín: Imprenta Oficial.

Canguilhem, G. (1983). Lo normal y lo patológico. Argentina: Siglo XXI.

Congreso de la República de Colombia (1890). Ley 123 sobre casas de corrección de menores y Escuelas de Trabajo. En: Consejo de Estado. Leyes colombianas de 1890. Bogotá: Imprenta La Luz.

Congreso de la República de Colombia (1920). Ley 98 de 1920, "Por la cual se crean Juzgados de menores para que se ocuparan de la atención al menor con problemas de conducta". Sf.

Courmont, P. (1913). Manual de Patología General. Cit.: Cadavid, T. (1924). Discolía de la pubertad. Medellín: Imprenta Oficial.

Cruchet, J-R. (1908). Les arriérés scolaires. Publicación, Paris: Masson. Cit.: T. Cadavid \& D. Velásquez (1921), Informe de la Casa de Menores y Escuela de Trabajo. Medellín: Imprenta Oficial. Sala Antioquia U.de.A.

Decroly, O.\& Buyse, R. (1923). Les applications américaines de la psychologie à l'organisation humaine et à l'éducation. Bruxelles: Lamertin. Cit.: Cadavid, T. (1924). Discolía de la pubertad. Medellín: Imprenta Oficial. 
Decroly, O. \& Shepers (1923). Le sélecction des mieux doués. Brueselas. Cit.: Cadavid, T. (1924). Discolía de la pubertad. Medellín: Imprenta Oficial.

Díaz, J. (1987). La educación especial y sus acciones en Antioquia. Medellín: Secretaría de Educación y Cultura.

Foucault, M. (2003). Vigilar y castigar. El nacimiento de la prisión. Buenos Aires: Siglo XXI.

Gobernación de Antioquia (1920). Casa de Menores y Escuela de Trabajo. Compilación de disposiciones que rigen la materia. Medellín: Imprenta oficial.

Gobernación de Antioquia (1914). Decreto 1107, Disposiciones generales, Casa de Corrección de Menores y Escuela de Trabajo. Medellín: Imprenta Oficial. Sala Antioquia U.de.A.

Gobernación de Antioquia (1912). Secretaría de Gobierno Departamental. Memoria de Gobierno, I, 1386, 1911-1914. Medellín: La Secretaría, p. 97. Archivo Histórico de Antioquia. Cit.: Marín, Juan A. (1992). Reconstrucción histórica de la Escuela de Trabajo San José, 1914-1991. Medellín: Funlam.

Gobernación de Antioquia (1914). Secretaría de Gobierno Departamental. Medellín: La Secretaría, pp. 99-100. Cit.: Marín, J. (1992). Reconstrucción histórica de la Escuela de Trabajo San José, 1914-1991. Medellín: Funlam.

Gobernación de Antioquia (1921). Casa de Menores y Escuela de Trabajo. Medellín: Imprenta Oficial. Sala Antioquia, U.de.A.

Hernández, E., et al. (2003). De la Sección de Psicotecnia al Laboratorio de Psicometría: seis décadas de algo más que medición psicológica en Colombia. Avances en Medición, 1(1), 6-16.

Huertas, R. (1998). Clasificar y Educar. Historia natural y social de la deficiencia mental. Madrid: CSIC.

Jiménez, L.M. (1920). Nuestras razas decaen. Algunos signos de degeneración colectiva en Colombia y en los países similares. Memoria presentada al Tercer Congreso Médico Colombiano reunido en Cartagena en enero de 1918. Bogotá: Imprenta y Litografía de Juan Casis, 1920.

Marín, J.A. (1992). Reconstrucción histórica de la Escuela de Trabajo San José, 19141991. Medellín: Funlam.

Marquez, L.C. (1921). Memoria que el Ministro de Gobierno presenta al Congreso de 1921. Bogotá: Imprenta Nacional.

Martínez, M. (1992). Criminalidad y violencia en Antioquia. Medellín: Imprenta de El Espectador. (Trabalho original publicado em 1895).

Melo, J. (1988) (Edit.). Historia de Antioquia. Medellín: Suramericana de Seguros - Editorial Presencia Ltda., p. 299.

Morel, B. (1857). Traité des dégénérescences physiques, intellectuelles et morales de l'espèce humaine et des causes qui produisent ces variétés maladives. Cit.: Caponi, 


\section{HISTÓRIA DA PSIQUIATRIA}

Sandra. (2012). Loucos e degenerados. Uma genealogía da psiquitria ampliada. Manguinhos: Fiocruz.

Olano, R. (1917). Colombia Revista Semanal, 1(44), marzo 28. Sala Antioquia, Biblioteca Piloto. Marín, Juan A. 1992. Reconstrucción histórica de la Escuela de Trabajo San José, 1914-1991. Medellín: Funlam.

Oficina Sanitaria Panamericana. Sociedades e instituciones científicas de la América Latina. Washington D.C.: diciembre de 1939, Publicaciones mimeografiadas; 84, p. 38. Repositorio de la OPS/OMS: http://iris.paho.org/xmlui/handle/123456789/1092

Richard, G. (1911). Pédagogie expérimentale. Cit.: Cadavid, T.; Velásquez, D. (1921). Informe de la Casa de Menores y Escuela de Trabajo. Medellín: Imprenta Oficial. Sala Antioquia U.de.A.

Relator (12/02/1930). Está en pésimas condiciones el pabellón de locos de la ciudad. Cali, febrero 12.

Rodríguez, J.M. (1896). Contribución al estudio de las degeneraciones de evolución. Idiotez. (Tesis). Facultad de Medicina, Universidad Nacional de Colombia. Bogotá: Imprenta Medardo Rivas.

Stepan, N.L. (1991). The Hour of Eugenics. Race, Gender, and Nation in Latin America. London: Ithaca - Cornell University Press.

Sáenz, Jr.; Saldarriaga, Ó. \& Ospina, A. (1997). Mirar la infancia: pedagogía, moral y modernidad en Colombia, 1903-1946. Bogotá: Colciencias, U.de.A, FNC, Uniandes, T. I, p. 55

Toro, G. (1924). Conceptos. In: Cadavid, T. Discolía de la pubertad. Medellín: Imprenta Oficial, p. 3.

Uribe, L. (1940). Manicomio Departamental, Informe del Director. Secretaría de Higiene y Asuntos Sociales, Capítulo VIII, pp. 104-112. Archivo Histórico de Antioquia.

\section{Resumos}

(Ortopedia da Alma. Degeneracionismo e Higiene Mental na Casa de Correção de Menores e Escola de Trabalho San Jose, Colômbia, 1914-1947)

Objetivo: Compreender as práticas e a formação experimental da psicopedagogia da infância anormal na Colômbia, realizadas na Casa de Correção de Menores e Escola de Trabalho San José, entre 1914 e 1947. Métodos e materiais: estudo qualitativo, de abordagem histórico-hermenêtica, mediante pesquisa documental, heurística de arquivos de patrimônio documental e historiografia institucional do período de estudo.

Conclusões: $O$ surgimento de um novo regime de educação especial (médico e psicopedagógico) estabeleceu uma relevante mudança do modelo de reformatório 
de castigo ao de intervenção pedagógica, influenciada pelo cruzamento de doutrinas científicas de fins do século XIX e inícios do século $X X$, como a regra do normal e do patológico, a teoria da degeneração e a eugenia, para o aperfeiçoamento de um sujeito moderno capaz de ser útil a si mesmo e à sociedade.

Palavras-chave: Correção de menores, infância anormal, degeneração, psicotécnica

(The orthopedics of the Soul. Degeneration and Mental Hygiene in the San Jose Juvenile Correctional Facility and Work School, Colombia 1914-1947)

Objective: To understand the practices and the experimental formation of the psychopedagogy of abnormal childhood in Colombia, carried out in the San Jose Juvenile Correctional Facility and Work School, Colombia, between 1914 and 1947. Methods and materials: qualitative study, of a historical-hermeneutic approach, using documentary research, and file heuristics of document heritage and institutional historiography within the study period.

Conclusions: The emergence of a new special education regime (medical and psychopedagogical) established an important change in the model of punishing reformatory to pedagogical intervention, influenced by crossing scientific doctrines of the late nineteenth and early twentieth centuries, such as the rule of normal and pathological, the degeneration theory and eugenics, for perfecting a modern subject who can be useful to itself and to society.

Key words: Correction of minors, abnormal childhood, degeneration, psychotechnology

(Orthopédie de l'âme. Dégénerationisme et hygiène mentale à la Maison de correction pour mineurs et de l〉École de travail San José, Colombie, 1914-1947)

Objectif: Comprendre les pratiques et la formation expérimentale de la psychopédagogie de l'enfance anormale en Colombie, réalisées à la Maison de correction et l'École de travail San José, entre 1914 et 1947. Méthodes et matériaux : étude qualitative, approche historique-herméneutique, par le biais d'une recherche documentaire, heuristique de fichiers de patrimoine documentaire et historiographie institutionnelle de la période d'étude.

Conclusions: L'émergence d'un nouveau régime d'éducation spécialisée (médicale et psychopédagogique) a établi un changement pertinent du modèle de maison de correction à celui d'intervention pédagogique, influencé par le croisement de doctrines scientifiques de la fin du XIXe et début du XXe siècles, comme la règle du normal et du pathologique, la théorie de la dégénérescence et l'eugénisme, pour le perfectionnement d'un sujet moderne capable d'être utile à lui-même et à la société.

Mots clés: Correction des mineurs, enfance anormale, dégénérescence, psychotechnique 
(Orthopädie der Seele. Degenerationstheorie und Mentale Hygiene in der Jugendstrafanstalt / Ausbildungszentrum San José, Kolumbien, 1914-1947)

Ziel: die Praxis und das experimentelle Ausbildungsprogram der Psychopädagogik der abnormalen Kindheit in Kolumbien zu untersuchen, am Fall der Jugendstrafanstalt / Ausbildungszentrum San José, von 1914 bis 1947. Methoden und Materialien: qualitative Studie, historisch-hermeneutischer Ansatz, dokumentarische Forschung, Heuristik der Dokumentdatei und der institutionellen Geschichtsschreibung innerhalb des Untersuchungszeitraumes.

Schlussfolgerungen: die Entstehung einer neuen (medizinischen und psycho-pädagogischen) Regelung im Bereich der Sonderpädagogik etabliert eine wichtige Wandlung vom Model der 'bestrafenden Erziehungsanstalt' zu jenem der pädagogischen Intervention. Diese Wandlung entsteht durch die Fusion wissenschaftlicher Lehren des späten neunzehnten und frühen zwanzigsten Jahrhundert, wie z.B. die Regel des normalen und des pathologischen, die Degenerationstheorie und die Eugenik. Sie wurde zur Perfektionierung des modernen Subjekts benutzt, das dadurch für sich selbst und für die Gesellschaft von Nutzen wird.

Stichwörter: Korrektur von Minderjährigen, abnorme Kindheit, Degeneration, psychotechnik

（1914-1947年哥伦比亚未成年人矫正所和圣胡安工读学校里的精神病治 疗, 退化和精神健康)

本论文的目的了解1914-1947年哥伦比亚的未成年人矫正所和圣胡安工读 学校在异常行为青少年教育心理学方面的试验和实践。研究方法和材料: 对事 件的质的分析, 从史学和方法论角度, 对该时期, 两个机构的档案资料和史料 进行详细的和整体全面的分析。结论是：在实践中产生了一个新的特殊教育制 度 (医疗和心理教育), 青少年矫正模式也产生了重要的变化, 由原来的以惩罚 为主的感化院做法, 转变为以教育为主的模式, 这种转变是由于十九世纪末二 十世纪初科学思想和社会思潮的转变, 特别是鉴定正常和病态的规则的变化, 有关人类退化和优生的理论的传播, 产生这些改进和转变的目的是为了培养一 种有益于个人，有益于社会的现代人格。

关键：未成年人的矫正，异常的童年，退化，心理分析技术。

Citação/Citation: Avendaño, J.G.; Ramirez, L.M.S. (2016, março). Ortopedia del alma. Degeneracionismo e higiene mental en la Casa de Corrección de Menores y Escuela de Trabajo San José, Colombia, 1914-1947. Revista Latinoamericana de Psicopatologia Fundamental, 19(1), 150-166.

Editores do artigo/Editors: Profa. Dra. Ana Maria G. Raimundo Oda e Prof. Dr. Paulo Dalgalarrondo. 
Recebido/Received: 15.7.2015/ 7.15.2015 Aceito/Accepted: 18.11.2015 / 11.18.2015

Copyright: (C) 2009 Associação Universitária de Pesquisa em Psicopatologia Fundamental/ University Association for Research in Fundamental Psychopathology. Este é um artigo de livre acesso, que permite uso irrestrito, distribuição e reprodução em qualquer meio, desde que o autor e a fonte sejam citados / This is an open-access article, which permits unrestricted use, distribution, and reproduction in any medium, provided the original authors and sources are credited.

Financiamento/Funding: Os autores declaram não terem sido financiados ou apoiados / The authors have no support or funding to report.

Conflito de interesses/Conflict of interest: Os autors declaram que não há conflito de interesses / The authors have no conflict of interest to declare.

\section{Jairo Gutiérrez Avendaño}

Doctorando en Ciencias Humanas y Sociales, Universidad Nacional de Colombia; Magíster en Educación, Universidad de Medellín; Filósofo, Universidad de Antioquia; Profesor e investigador adscrito al grupo de Estudios de Fenómenos Psicosociales, Fundación Universitaria Luis Amigó.

Calle 74 N 72B - 110, Apto. 2408 - Almendra

Medellín, Colombia

e-mail: jairo.gutierrezav@amigo.edu.co

\section{Lina Marcela Silva Ramírez}

Magíster en Historia y Socióloga, Universidad de Antioquia; Docente investigadora del Centro de Educación para el Desarrollo, Corporación Universitaria Minuto de Dios, Sede Bello, Antioquia.

Calle 74 N 72B - 110, Apto. 2408 - Almendra

Medellín, Colombia

e-mail: 1silvaramir@uniminuto.edu.co

\section{(cc) BY-NC}

This is an open-access article, which permits unrestricted use, distribution, and reproduction in any medium for non-commercial purposes provided the original authors and sources are credited. 